\title{
Comparison of the long-term efficacy and safety of generic Tacrobell with original tacrolimus (Prograf) in kidney transplant recipients
}

This article was published in the following Dove Press journal:

Drug Design, Development and Therapy

12 January 2017

Number of times this article has been viewed

\author{
Seung Yeon Son' \\ Hye Ryoun Jang' \\ Jung Eun Lee' \\ Heejin Yoo ${ }^{2}$ \\ Kyunga Kim ${ }^{2,3}$ \\ Jae Berm Park ${ }^{4}$ \\ Sung Joo Kim ${ }^{4}$ \\ $\mathrm{Ha}$ Young $\mathrm{Oh}^{1}$ \\ Wooseong Huh' \\ 'Department of Medicine, Samsung \\ Medical Center, Sungkyunkwan \\ University School of Medicine, \\ ${ }^{2}$ Statistics and Data Center, Research \\ Institute for Future Medicine, Samsung \\ Medical Center, ${ }^{3}$ Department of \\ Digital Health, SAIHST, ${ }^{4}$ Department \\ of Surgery, Samsung Medical Center, \\ Sungkyunkwan University School of \\ Medicine, Seoul, Republic of Korea
}

Correspondence: Wooseong Huh Department of Medicine, Samsung Medical Center, 8I Irwon-ro, Gangnam-gu, Seoul 0635I,

Republic of Korea

$\mathrm{Tel}+82234103443$

Fax +82 234100064

Email wooseong.huh@samsung.com

\begin{abstract}
This study aimed to evaluate the long-term efficacy and safety of a generic tacrolimus (Tacrobell [TCB]) compared to the original tacrolimus (Prograf [PGF]) in kidney transplant recipients. In this retrospective observational study, we analyzed the data from 444 patients who took TCB as a first-line immunosuppressive drug and 245 patients who took PGF. The 5-year graft survival rate was $92 \%$ for patients in the PGF group and $97 \%$ for patients in the TCB group, respectively. Cox proportional hazards for a one-sided, noninferiority model showed noninferiority (upper confidence interval [CI] limit of the hazard ratio $[\mathrm{HR}]<1.2$ ) for TCB compared to PGF (HR: 0.58; 95\% CI: 0-1.14). The 5-year patient survival rate was 96\% for patients in the PGF group and $97 \%$ for patients in the TCB group. Cox proportional hazards for a one-sided, noninferiority model showed noninferiority (upper confidence interval limit of the HR<2.0) for TCB compared to PGF (HR: 0.83; 95\% CI: 0-1.95). The 5-year acute rejection-free graft survival rate was not significantly different between the groups (TCB 67\%, PGF $68.8 \% ; P=0.6286$ ). The incidence of adverse events including adverse cardiovascular or cerebrovascular events, malignancies, new-onset diabetes after transplantation, and infection events did not differ significantly between the two groups. We conclude that TCB is a comparable alternative to the original tacrolimus as a first-line immunosuppressive drug. Producers of generics should support further study of their products after approval to assure physicians of their efficacy and safety.
\end{abstract}

Keywords: kidney transplant, generic, tacrolimus

\section{Introduction}

Tacrobell (TCB; Chong Kun Dang Pharmaceutical Corp, Seoul, Republic of Korea) is a generic formulation of tacrolimus that was approved in 2004 by the Republic of Korea Ministry of Food and Drug Safety (KMFDS). ${ }^{1}$ The criteria for approval of a generic formulation by the KMFDS are similar to those of the US Food and Drug Administration or the European Medicinal Agency. The KMFDS requires the manufacturer to conduct a bioequivalence study in healthy volunteers as a clinical trial..$^{2-4}$

Generic formulation has the potential for cost savings. On the one hand, in a country that has an adequate public health care system, drug cost saving is an important component for maintaining comprehensive and equitable health care. ${ }^{5}$ On the other hand, if comprehensive health insurance coverage is not available, a lower drug cost may improve the compliance of patients who have to take long-term medication, such as immunosuppressants. $^{6}$

Although there are benefits from using a generic formulation, the generic substitution of drugs with a narrow therapeutic index, such as immunosuppressants, is a hotly 
debated issue. A main concern about using generic immunosuppressant drugs is whether a demonstration of bioequivalence in healthy volunteers offers a sufficient guarantee of therapeutic equivalence in the transplant recipients. ${ }^{7-12}$ The transplantation societies have claimed that they need more clinical information to be convinced of the safety of generic immunosuppressants.

Samsung Medical Center Organ Transplantation Center has used the original tacrolimus, Prograf (Astellas Pharma Inc, Tokyo, Japan) since 2000 and the generic tacrolimus since 2007. Based on the long-term experience of the two drugs, we conducted a retrospective observational study to evaluate the long-term efficacy and safety of the generic tacrolimus compared to the original tacrolimus in kidney transplant $(\mathrm{KT})$ recipients.

\section{Materials and methods Study design and participants}

The present retrospective observational study was approved by the institutional review board of Samsung Medical Center (approval no SMCIRB 2014-10-046); the process of patient consent was waived.

A total of 1,319 adult ( $\geq 18$ years old) patients received a KT between January 1, 2000 and June 30, 2013 at the Samsung Medical Center, Seoul, Republic of Korea. Among these, 603 patients took the generic tacrolimus (TCB), and 369 patients took the original tacrolimus (PGF) as a first-line immunosuppressive drug. We excluded patients in each group according to the following criteria: multiple organ transplants
(TCB, 37 patients; PGF, 17 patients), KT after desensitization (TCB, 66 patients), immunological tolerance to KT (TCB, 1 patient), and conversion from each drug. Among the patients who converted from TCB to PGF (55 patients), 40 participated in clinical trials using TCB as a study drug and converted to PGF at the end of the study. In the other 15 patients, we cannot find a specific reason for conversion in the medical records. Among the patients who converted from PGF to TCB (107 patients), 80 were enrolled in conversion clinical trials. In the other 27 , we could not find a specific reason in the medical records. Ultimately, we analyzed the data from 444 patients who were administered TCB and 245 patients who were administered PGF (Figure 1). Follow-up continued until June 30, 2014.

\section{Data collection}

We collected the data from the medical records and the database of the Organ Transplantation Center, Samsung Medical Center. The recipient variables included age, sex, body mass index (BMI), cause of end-stage renal disease, type and duration of renal replacement therapy, history of previous $\mathrm{KT}$, cytomegalovirus (CMV) serology, and hepatitis B and C virus infection status. The donor variables included age, sex, BMI, serum creatinine level of the donor, CMV serology, and the donor-recipient relationship. The transplantation-related variables included peak panel-reactive antibody (PRA) levels, number of human lymphocyte antigen (HLA) mismatches, cold ischemic time (CIT), and immunosuppressive induction and maintenance medications. Delayed graft function was defined as the need for dialysis in the first week after KT.

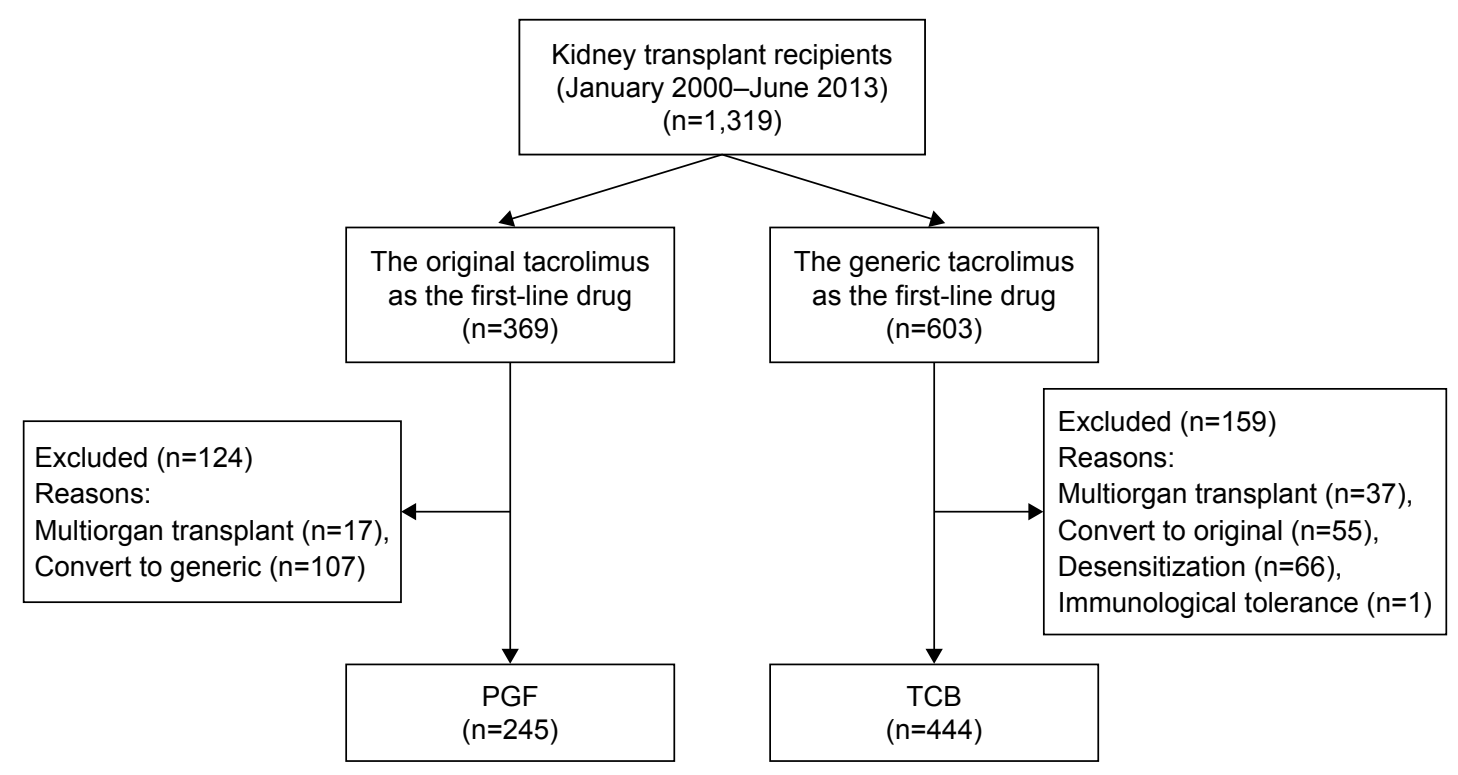

Figure I Patient disposition.

Abbreviations: PGF, Prograf; TCB, Tacrobell. 
Primary nonfunction was defined as permanent loss of allograft function starting immediately after KT. Acute rejection (AR) was defined clinically by an acute deterioration in allograft function and confirmed with tissue diagnosis. Banff borderline AR was not considered as an AR episode. Graft failure was defined as transplant nephrectomy, retransplantation, or return to long-term dialysis. We evaluated the complications after KT, such as cardiovascular events, cerebrovascular events, infection, malignancy, and new-onset diabetes after transplantation (NODAT). The cardiovascular events included myocardial infarction, symptomatic coronary artery disease requiring percutaneous intervention or coronary artery bypass grafting, ischemic heart disease (excluding normal coronaries in coronary angiography), and congestive heart failure. The cerebrovascular events included ischemic stroke (transient ischemic accident, cerebral infarction), and cerebral hemorrhage. Diagnosis of malignancy required documented histopathology. NODAT was defined as a fasting plasma glucose level of $>126 \mathrm{mg} / \mathrm{dL}$ on at least two different dates, or a condition requiring oral hypoglycemic agents or insulin for glycemic control.

\section{Immunosuppressive regimens}

From 2000 to 2013, the immunosuppressive protocol in the transplantation center was revised gradually. A remarkable change was the introduction of induction therapy. Induction therapy with rabbit antihuman thymocyte globulin (antithymoglobulin) or basiliximab was used routinely in 2005. Essentially, a triple maintenance immunosuppressive regimen comprising calcineurin inhibitor, mycophenolate mofetil, and corticosteroids was used. The generic or original tacrolimus was administered orally as two separate doses, starting at $0.15 \mathrm{mg} / \mathrm{kg} / \mathrm{day}$. The doses of the two drugs were adjusted to attain the same guideline target trough level. Target trough levels of tacrolimus were $12-15 \mathrm{ng} / \mathrm{mL}$ during the second postoperative week, $10-12 \mathrm{ng} / \mathrm{mL}$ within 1 month of transplantation, $8-10 \mathrm{ng} / \mathrm{mL}$ within 3 months of transplantation, and 6-8 ng/mL thereafter. Mycophenolate mofetil was administered at a dose of $1,500 \mathrm{mg} /$ day to patients of both groups except for those in whom it provoked side effects. Five hundred milligrams of methylprednisolone (MPD) was administered intravenously before and during surgery, and on the first postoperative day, and MPD was gradually tapered to a minimal dose thereafter.

\section{End points}

The primary end points were the 5-year patient survival and graft survival. The secondary end points were the 5-year
AR-free graft survival and the incidence of complications including cardiovascular event, cerebrovascular event, infection, malignancy, and NODAT.

\section{Statistical analyses}

The characteristics of the two groups were analyzed. Continuous variables are expressed as the mean \pm standard deviation or median (interquartile range) and were compared using a two-sided unpaired two-sample $t$-test. A Mann-Whitney $U$-test was used if data were not normally distributed. Categorical variables are expressed as a number (\%) and were analyzed using a chi-square or Fisher's exact test.

The patient and graft survival rate and AR-free graft survival rate of the two groups were plotted using a KaplanMeier method. To compare the patient and graft survival rates of the two groups, a Cox proportional-hazards model noninferiority test was conducted. The association of risk factors with patient and graft survival rates was identified using a Cox proportional-hazards model in multivariable analyses. In case of a rare event, a Cox proportional-hazards model using Firth's penalized maximum likelihood estimation method was applied. Variables with $P<0.05$ in the univariable analysis were included in the multivariable analysis. AR events were included in the Cox models as time-dependent variables during follow-up. The 5-year AR-free graft survival rates in the two groups were evaluated using the counting process method for recurrent events data. ${ }^{13}$ The association of risk factors with $\mathrm{AR}$ was identified by the robust sandwich estimate of Lin and Wei for the covariance matrix. ${ }^{14}$ Variables with $P<0.1$ in the univariable analysis were included in the multivariable analysis.

The incidence of adverse cardiovascular or cerebrovascular events, malignancy, and NODAT after KTs was compared using a Fisher's exact test. The distribution of infections after KTs was analyzed using a chi-square test for clustered data. ${ }^{15}$ The $P$-values were corrected using a Bonferroni post hoc method in case of multiple testing. $P<0.05$ was considered to be significant. Statistical analyses were conducted using SAS version 9.4 (SAS Institute Inc, Cary, NC, USA).

\section{Results \\ Study population}

Demographic characteristics of the patient population studied are listed in Table 1 . There were many changes in the policies and procedures for solid organ transplants over 13 years. Introduction of induction therapy as mentioned above, using expanded criteria donors, and increasing the number of deceased donor KTs were significant changes. 
Table I Demographic and clinical characteristics

\begin{tabular}{|c|c|c|c|}
\hline Variable & PGF group $(n=245)$ & TCB group $(n=444)$ & $P$-value \\
\hline \multicolumn{4}{|l|}{ Recipient } \\
\hline Age, year & $39.8 \pm 10.1$ & $45.8 \pm 11.2$ & $<0.0001$ \\
\hline Male sex, n (\%) & $157(64.1)$ & $258(58.1)$ & 0.1251 \\
\hline BMI, kg/m² & $22.7 \pm 3.5$ & $22.7 \pm 3.4$ & 0.9310 \\
\hline Cause of ESRD, $\mathrm{n}(\%)$ & & & 0.0483 \\
\hline Diabetes mellitus & $21(8.6)$ & $63(14.2)$ & \\
\hline IgA nephropathy & $24(9.8)$ & $62(14.0)$ & \\
\hline FSGS & $6(2.5)$ & $5(1.1)$ & \\
\hline Other glomerulonephritis & 39 (I5.9) & $51(11.5)$ & \\
\hline ADPCK & $8(3.3)$ & $21(4.7)$ & \\
\hline Others & $8(3.3)$ & $18(4.1)$ & \\
\hline Unknown & $139(56.7)$ & $224(50.5)$ & \\
\hline HBsAg+, n (\%) & II (4.5) & $23(5.2)$ & 0.6888 \\
\hline Anti-HCV Ab+, n (\%) & $6(2.5)$ & $5(1.1)$ & 0.2113 \\
\hline CMV-, n (\%) & I (0.4) & $0(0)$ & 0.3556 \\
\hline Dialysis duration, months & $24.4(5.1-65.3)$ & $32.2(2.8-72.7)$ & 0.7710 \\
\hline Dialysis modality, n (\%) & & & 0.8886 \\
\hline Preemptive & $23(9.4)$ & $66(14.9)$ & \\
\hline Hemodialysis & I75 (7I.4) & $308(69.3)$ & \\
\hline CAPD & $47(19.2)$ & $70(15.8)$ & \\
\hline PRA > 30\%, n (\%) & $14(5.7)$ & $50(11.3)$ & 0.0163 \\
\hline Secondary KT, n (\%) & $22(9)$ & $36(8.1)$ & 0.6816 \\
\hline \multicolumn{4}{|l|}{ Donor } \\
\hline Age, year & $40.7 \pm 12.5$ & $44.0 \pm 12.7$ & 0.0010 \\
\hline BMI, kg/m² & $23.5 \pm 3.0$ & $23.4 \pm 3.6$ & 0.5592 \\
\hline Relationship, n (\%) & & & 0.0004 \\
\hline Living & I84 (75.I) & $274(6 \mid .7)$ & \\
\hline Deceased & 61 (24.9) & $170(38.3)$ & \\
\hline No of HLA mismatches & $3.9 \pm 1.5$ & $3.3 \pm 1.5$ & $<0.000 \mathrm{I}$ \\
\hline \multicolumn{4}{|l|}{ Transplant } \\
\hline Cold ischemic time, $\min$ & $59.0(45.0-144.0)$ & $100.0(66.0-224.0)$ & $<0.000 \mathrm{I}$ \\
\hline Induction therapy, n (\%) & $76(31.0)$ & $433(97.5)$ & $<0.0001$ \\
\hline Delayed graft function, n (\%) & $30(12.2)$ & $48(10.8)$ & 0.5696 \\
\hline Primary nonfunction, n (\%) & $4(1.6)$ & $4(0.9)$ & 0.4636 \\
\hline Acute rejection, n (\%) & $92(37.6)$ & $126(28.4)$ & 0.0132 \\
\hline Repeated acute rejection, $\mathrm{n}(\%)$ & $27(\mathrm{II} .0)$ & $36(8.1)$ & 0.2042 \\
\hline
\end{tabular}

Notes: Continuous variables are expressed as the mean \pm standard deviation or median (interquartile range). Categorical variables are expressed as number (\%).

Abbreviations: ADPCK, autosomal dominant polycystic kidney; BMI, body mass index; CAPD, continuous ambulatory peritoneal dialysis; CMV, cytomegalovirus; ESRD, end-stage renal disease; FSGS, focal segmental glomerulosclerosis; HBsAg, hepatitis B surface antigen; HCV, hepatitis C virus; HLA, human leukocyte antigen; KT, kidney transplant; min, minutes; PGF, Prograf; PRA, panel reactive antibody; TCB, Tacrobell.

Therefore, the characteristics of each group were different. Patients in the TCB group were more likely to be older than those in the PGF group $(P<0.0001)$. The distributions of primary cause of end-stage renal disease were different in both groups ( $P=0.0483)$. The age of the donor at the time of KT was higher in the TCB group $(P=0.0010)$. The proportion of deceased donors was higher in the TCB group $(P=0.0004)$. The number of HLA mismatches was higher in the TCB group $(P<0.0001)$. The percentage of peak or current PRA above $30 \%$ was higher in the TCB group $(P=0.0163)$. CIT was longer in the TCB group $(P<0.0001)$. Induction therapy with antithymoglobulin or basiliximab was used for the majority of patients taking TCB $(97.5 \%)$ and for only $31 \%$ of those taking PGF $(P<0.0001)$. The incidence of delayed graft function and primary nonfunction was similar in each group ( $P=0.5696$ and $P=0.4636$, respectively). The incidence of AR was lower in the TCB group $(P=0.0132)$. The proportion of patients who had experienced repeated AR was similar in each group $(P=0.2042)$.

\section{Five-year graft survival and patient survival}

The 5-year graft survival rate was $92 \%$ for patients in the PGF group and $97 \%$ for patients in the TCB group (Figure 2), but the difference was not significant $(P=0.1857)$ after adjusting for other risk factors. Furthermore, a significant 


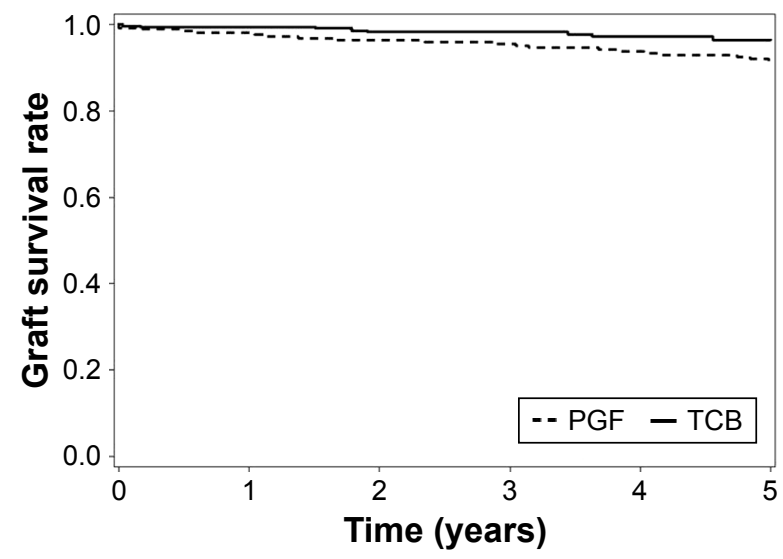

Figure 2 Five-year graft survival rates.

Notes: The solid line indicates graft survival in the TCB group patients; the broken line indicates graft survival in the PGF group patients. The 5-year graft survival rate was $92 \%$ for patients in PGF and $97 \%$ for patients in TCB, but the difference was not significant $(P=0.1857)$ after adjusting for other risk factors. A significant noninferiority (with a margin of 0.2 ) of TCB was observed compared to PGF (HR: 0.58; 95\% Cl: 0-I.I4).

Abbreviations: $\mathrm{Cl}$, confidence interval; HR, hazard ratio; PGF, Prograf; TCB, Tacrobell.

noninferiority (with a margin of 0.2 ) of TCB was observed when compared to PGF (HR: 0.58; 95\% CI: 0-1.14). The risk factors for graft failure were hepatitis $\mathrm{C}$ virus infection (HR: 8.44 ; 95\% CI: 2.46-28.95), primary nonfunction (HR: 6.20; 95\% CI: $1.90-20.28$ ), and AR (HR: $2.74 ; 95 \%$ CI: $1.43-5.26)$.

The 5-year patient survival rate was $96 \%$ for patients in the PGF group and $97 \%$ for patients in the TCB group (Figure 3), but the difference between the groups was not significant $(P=0.7143)$ after adjusting for other risk factors.

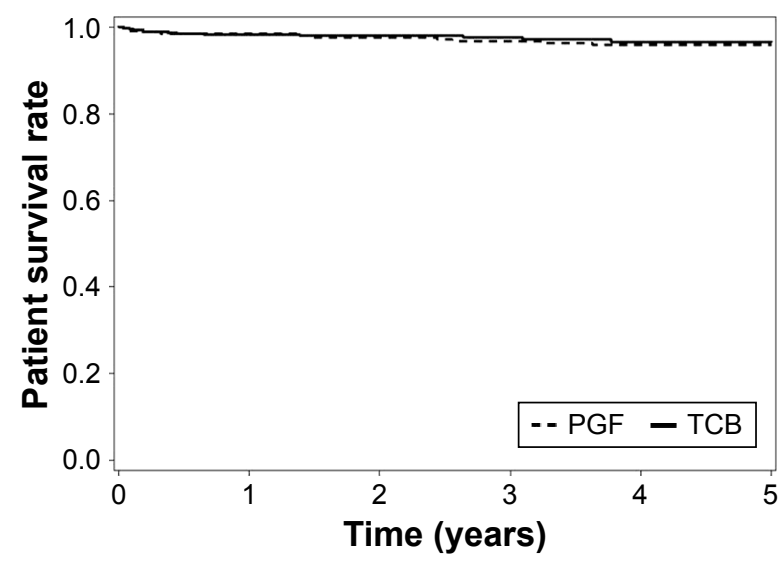

Figure 3 Five-year patient survival rates.

Notes: The solid line indicates survival of patients in the TCB group; the broken line indicates survival of patients in the PGF group. The 5 -year patient survival rate was $96 \%$ for patients in the PGF group and $97 \%$ for patients in the TCB group (Figure 3), but the difference was not significant $(P=0.7143)$ after adjusting for other risk factors. A significant noninferiority (with a margin of I) of TCB was observed compared to PGF (HR 0.83; 95\% Cl: 0-1.95).

Abbreviations: $\mathrm{Cl}$, confidence interval; $\mathrm{HR}$, hazard ration; PGF, Prograf; TCB, Tacrobell.

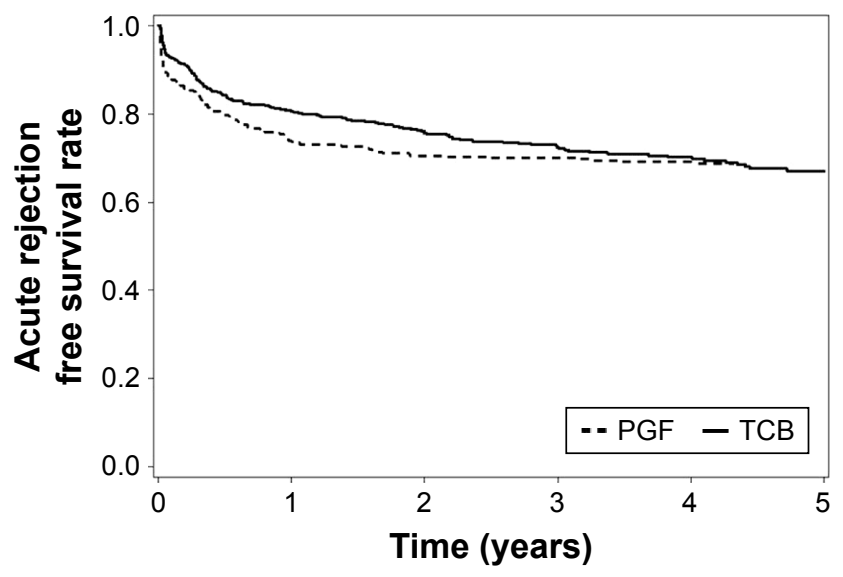

Figure 4 Five-year acute rejection-free graft survival rates.

Notes: The solid line indicates acute rejection-free graft survival in the TCB group; the broken line indicates acute rejection-free graft survival in the PGF group. The 5 -year acute rejection-free graft survival rate was not significantly different between the groups (TCB 67\%, PGF 68.8\%; $P=0.6286$ ).

Abbreviations: PGF, Prograf; TCB, Tacrobell.

A significant noninferiority (with a margin of 1 ) of TCB was observed when compared to PGF (HR: 0.83 ; 95\% CI: $0-1.95)$. The risk factors for patient survival were older recipient age (HR: 1.08; 95\% CI: 1.03-1.13), longer dialysis duration (HR: $1.01 ; 95 \%$ CI: 1.00-1.02), delayed graft function (HR: $3.00 ; 95 \%$ CI: 1.21-7.23), and repeated AR (HR: 5.06; 95\% CI: 1.66-15.46).

\section{Five-year AR-free graft survival rate}

There was no significant difference in the 5-year AR-free graft survival rate between the groups (TCB 67\%, PGF $68.8 \% ; P=0.6286$ ) (Figure 4). In multivariable analysis, no induction therapy (HR: $1.45 ; 95 \%$ CI: $1.00-2.10$ ), older donor age (HR: $1.01 ; 95 \%$ CI: $1.00-1.02$ ), and hepatitis B virus positive recipient (HR: 1.60; 95\% CI: 1.04-2.50) were associated with the development of AR.

\section{Adverse events}

The incidence of adverse cardiovascular events ( $P=0.1793)$, adverse cerebrovascular events $(P=0.2703)$, malignancies $(P=0.7870)$, NODAT $(P=0.9)$, and infection events $(P=0.3060)$ did not differ significantly between the groups (Table 2).

\section{Discussion}

The present study showed that there was no difference in long-term graft and patient survival between those taking TCB or PGF, although the incidence of AR was lower in those taking TCB. In multivariable analysis, there was no group effect. We also found that AR-free survival rates were similar 
Table 2 Long-term adverse events

\begin{tabular}{llll}
\hline Adverse event & PGF group (n=245) & TCB group (n=444) & P-value \\
\hline Cardiovascular events, n (\%) & $6(2.5)$ & $4(0.9)$ & 0.1793 \\
Cerebrovascular events, n (\%) & $7(2.9)$ & $7(1.6)$ & 0.2703 \\
Malignancy, n (\%) & $6(2.5)$ & $9(2.0)$ & 0.7870 \\
NODAT, n (\%) & $28(11.4)$ & $49(11.1)$ & 0.9000 \\
Infection events, n (\% of total infection events) & & & 0.3060 \\
UTI & $46(13.6)$ & $85(17.5)$ & $29(6.0)$ \\
Pneumonia & $19(5.6)$ & $0(0.0)$ & $204(41.9)$ \\
PJP & $2(0.6)$ & $169(34.7)$ & \\
CMV infection & $139(41.0)$ & $133(39.2)$ & \\
Others & & \\
\hline
\end{tabular}

Abbreviations: CMV, cytomegalovirus; NODAT, new-onset diabetes after transplantation; PGF, Prograf; PJP, Pneumocystis jiroveci pneumonia; TCB, Tacrobell; UTI, urinary tract infection.

between the two groups. The incidence of adverse events such as adverse cardiovascular or cerebrovascular events, malignancies, NODAT, and infection events did not differ significantly between the two groups. Although we decided to exclude the patients who converted from each drug, we planned to determine the reason for conversion when analyzing adverse events. However, the majority of the patients converted to participate in clinical trials, and we could not find specific reasons for conversion in the medical records of the other patients. Therefore, we did not eventually evaluate the data of the patients who converted from each drug.

A main concern of prescribing generic immunosuppressant drugs is whether a demonstration of bioequivalence using a single-dose study in healthy adults is sufficient evidence of therapeutic equivalence in patients with solid organ transplants. ${ }^{5,10}$ To assure physicians of the efficacy and safety of generic immunosuppressant drugs, the drug should be validated by prospective, multicenter, large-scale, double-blind, randomized, and long-term clinical trials. ${ }^{8,16}$ However, it is difficult to conduct such ideal clinical trials with generics because the time and cost of running clinical trials is a major portion of drug development. If a regulator requests a manufacturer to conduct clinical trials in patients to approve a generic, then cost savings - the main purpose of using generics - cannot be achieved.

In the transplant community, there are some requests for generic companies to support further research such as pharmacokinetic studies in transplant recipients and investigator-initiated clinical trials for enrichment of the drug information. Postmarketing surveillance studies are also recommended to obtain additional safety data. ${ }^{10}$

For the generic tacrolimus (TCB), two investigator-initiated and generic company-supported clinical trials have been conducted. One trial was a short-term, single-arm, multicenter study. ${ }^{17}$ Although the study was not comparative and enrolled a small number of subjects, it showed that the AR rate in 6 months was $10.6 \%$ (95\% CI: 4.4\%-16.9\%). There were no unexpected serious adverse events. The other trial was a pharmacokinetic study in KT recipients to compare the pharmacokinetic parameters of the original and the generic tacrolimus. ${ }^{18}$ This study showed that the generic tacrolimus had an earlier and higher $\mathrm{C}_{\max }$ than the original tacrolimus. However, dose-adjusted $\mathrm{AUC}_{0-12}$ was not different, and 9-month efficacy and safety data were comparable. The present study showed the results of a long-term observational study.

There were some limitations to this study. 1) As this study was retrospective, there is a possibility of unknown or unmeasured confounding variables. 2) There were several significant differences between the two groups including the number of HLA mismatches, the proportion of patients with induction therapy, the proportion of deceased donors, CIT, the age of recipient and donor, and the period of observation. To mitigate the influence of these factors, Cox proportional-hazards models were applied to adjust both for confounding variables and for differences in length of follow-up. Although there were some limitations because of the retrospective and singlecentered nature of the study, the number of enrolled subjects was relatively large $(n=689)$, and the period of follow-up was sufficient to evaluate late complications. Therefore, the present study provides additional long-term efficacy and safety data for the generic tacrolimus.

The present study shows comparable outcomes for the generic tacrolimus in a de novo setting. We cannot extrapolate the acceptable outcomes in conversion settings from the results of the present study. The generic tacrolimus has not shown equivalent pharmacokinetic parameters in $\mathrm{KT}$ recipients. Even in stable patients with a low risk of $A R$, more frequent visits to the clinic for monitoring should be required until the trough levels of tacrolimus concentration stabilize. , $^{8,20}$ 
Nonadherence to medication by transplant recipients is a risk factor for poor graft outcome. ${ }^{21-23}$ High medication costs have been identified as a risk factor for nonadherence in countries that have inadequate health insurance coverage. ${ }^{22-24}$ In these countries, using adequately tested bioequivalent generic medications may be helpful for improving graft outcome and saving costs. However, it will be complicated to analyze the effects of using generic medications in countries that have adequate health insurance coverage. Although the use of generic immunosuppression may reduce drug costs, pharmacoeconomic analyses must also take into account added patient care costs for increased monitoring, clinic visits, patient education, medication reconciliation, and communication with pharmacies. ${ }^{10}$

\section{Conclusion}

TCB is an alternative comparable to the original tacrolimus as a first-line immunosuppressive drug. However, the results of the present study cannot be generalized to other generic formulations of tacrolimus. Uncontrolled substitutions from one generic to another must be avoided. ${ }^{25}$ Producers of generics should support further study of their products after approval to assure physicians of their efficacy and safety.

\section{Acknowledgment}

This study was supported by Chong Kun Dang Pharmaceutical, Corp., Seoul, Republic of Korea. Seung Yeon Son is currently working at Gimpo Woori Hospital, Gimpo, Republic of Korea.

\section{Disclosure}

The authors report no conflicts of interest in this work.

\section{References}

1. Drug approval information [website on the Internet]. Korea Ministry of Food and Drug Safety [cited July 1, 2016]. Available from: https:// ezdrug.mfds.go.kr. Accessed July 1, 2016.

2. Standard on bioequivalence study management. No. 2014-49 [website on the Internet]. [cited July 1, 2016]. Available from: https://www.mfds. go.kr. Accessed July 1, 2016.

3. Guidances (Drugs). [website on the Internet]. US Federal Drug Agency [cited July 1, 2016]. Available from: http://www.fda.gov/Drugs/ GuidanceComplianceRegulatoryInfromation/Guidances. Accessed July $1,2016$.

4. Guideline on the investigation of bioequivalence. [website on the Internet]. European Medicines Agency [cited 2016 July 1]. Available from: http://ema.europa.eu. Accessed July 1, 2016.
5. Molnar AO, Fergusson D, Tsampalieros AK, et al. Generic immunosuppression in solid organ transplantation: systematic review and meta-analysis. BMJ. 2015;350:h3163.

6. Jha V, Muthukumar T, Kohli HS, Sud K, Gupta KL, Sakhuja V. Impact of cyclosporine withdrawal on living related renal transplants: a singlecenter experience. Am J Kidney Dis. 2001;37(1):119-124.

7. Al Ameri MN, Whittaker C, Tucker A, Yaqoob M, Johnston A. A survey to determine the views of renal transplant patients on generic substitution in the UK. Transpl Int. 2011;24(8):770-779.

8. Alloway RR, Isaacs R, Lake K, et al. Report of the American Society of Transplantation conference on immunosuppressive drugs and the use of generic immunosuppressants. Am J Transplant. 2003; 3(10):1211-1215.

9. Banahan BF 3rd, Kolassa EM. A physician survey on generic drugs and substitution of critical dose medications. Arch Intern Med. 1997;157(18): 2080-2088.

10. Harrison JJ, Schiff JR, Coursol CJ, et al. Generic immunosuppression in solid organ transplantation: a Canadian perspective. Transplantation. 2012;93(7):657-665.

11. Sabatini S, Ferguson RM, Helderman JH, Hull AR, Kirkpatrick BS, Barr WH. Drug substitution in transplantation: a National Kidney Foundation White Paper. Am J Kidney Dis. 1999;33(2):389-397.

12. Uber PA, Ross HJ, Zuckermann AO, et al. Generic drug immunosuppression in thoracic transplantation: an ISHLT educational advisory. J Heart Lung Transplant. 2009;28(7):655-660.

13. Anderson PK, Borgan O, Gill RD, Keiding N. Statistical Models Based on Counting Processes. New York: Springer-Verlag; 1992.

14. Lin DY, Wei LJ. The robust inference for the proportional hazards model. J Am Stat Assoc. 1989;84:1074-1078.

15. Jung $\mathrm{SH}$, Kang $\mathrm{SH}, \mathrm{Ahn} \mathrm{C}$. Chi-square test for $\mathrm{R} \times \mathrm{C}$ contingency tables with clustered data. J Biopharm Stat. 2003;13(2):241-251.

16. Klintmalm GB. Immunosuppression, generic drugs and the FDA. Am J Transplant. 2011;11(9):1765-1766.

17. Kim SJ, Huh KH, Han DJ, et al. A 6-month, multicenter, single-arm pilot study to evaluate the efficacy and safety of generic tacrolimus (TacroBell) after primary renal transplantation. Transplant Proc. 2009;41(5): 1671-1674.

18. Min SI, Ha J, Kim YS, et al. Therapeutic equivalence and pharmacokinetics of generic tacrolimus formulation in de novo kidney transplant patients. Nephrol Dial Transplant. 2013;28(12):3110-3119.

19. McDevitt-Potter LM, Sadaka B, Tichy EM, Rogers CC, Gabardi S. A multicenter experience with generic tacrolimus conversion. Transplantation. 2011;92(6):653-657.

20. Momper JD, Ridenour TA, Schonder KS, Shapiro R, Humar A, Venkataramanan R. The impact of conversion from Prograf to generic tacrolimus in liver and kidney transplant recipients with stable graft function. Am J Transplant. 2011;11(9):1861-1867.

21. Butler JA, Roderick P, Mullee M, Mason JC, Peveler RC. Frequency and impact of nonadherence to immunosuppressants after renal transplantation: a systematic review. Transplantation. 2004;77(5): 769-776.

22. Figueiras MJ, Alves NC, Marcelino D, Cortes MA, Weinman J, Horne R. Assessing lay beliefs about generic medicines: development of the generic medicines scale. Psychol Health Med. 2009;14(3):311-321.

23. Fine RN, Becker Y, De Geest S, et al. Nonadherence consensus conference summary report. Am J Transplant. 2009;9(1):35-41.

24. Kasiske BL, Cohen D, Lucey MR, Neylan JF. Payment for immunosuppression after organ transplantation. American Society of Transplantation. JAMA. 2000;283(18):2445-2450.

25. van Gelder $\mathrm{T}$. What is the future of generics in transplantation? Transplantation. 2015;99(11):2269-2273. 


\section{Publish your work in this journal}

Drug Design, Development and Therapy is an international, peerreviewed open-access journal that spans the spectrum of drug design and development through to clinical applications. Clinical outcomes, patient safety, and programs for the development and effective, safe, and sustained use of medicines are the features of the journal, which

has also been accepted for indexing on PubMed Central. The manuscript management system is completely online and includes a very quick and fair peer-review system, which is all easy to use. Visit http://www.dovepress.com/testimonials.php to read real quotes from published authors.

Submit your manuscript here: http://www.dovepress.com/drug-design-development-and-therapy-journal 